\title{
Extended stellar kinematics of elliptical galaxies in the Fornax cluster $^{\star, \star \star}$
}

\author{
A.W. Graham ${ }^{1}$, M.M. Colless ${ }^{1}$, G. Busarello ${ }^{2}$, S. Zaggia ${ }^{2}$, and G. Longo ${ }^{2}$ \\ 1 Mount Stromlo and Siding Spring Observatories, Australian National University, PMB Weston Creek PO, ACT 2611, Australia \\ 2 Osservatorio Astronomico di Capodimonte, via Moiariello 16, I-80131 Napoli, Italy
}

Received February 4; accepted June 17, 1998

\begin{abstract}
We present extended stellar kinematics for a sample of elliptical galaxies in the Fornax cluster. Out of the 13 galaxies presented here, five (FCC 119, FCC 136, NGC 1373, NGC 1428, FCC 335) have no previously published kinematical data. Major-axis velocity dispersion profiles (VDPs) and rotation curves (RCs) are given for 12 of the galaxies. A major feature of this data is the spatial extension: for 8 galaxies the data extends beyond $1 R_{\mathrm{e}}$, and for 5 it extends beyond $2 R_{\mathrm{e}}$. Compared to the previously available data, this corresponds to an increase in spatial coverage by a factor from 1 to 5 . The present sample represents $86 \%$ of the ellipticals in Fornax brighter than $B_{\mathrm{T}}=15$ mag.

Five of the ellipticals in the sample turn out to be rotationally-supported systems, having positive rotation parameter $\log \left(\frac{V}{\sigma}\right)^{*}$. One of these five, and another 3 galaxies from the remaining sample, display evidence for bar-like kinematics.

The data indicate that the true number of "dynamically hot" stellar systems, is much lower than previously thought: of the Es in the present sample only 1/4 are confirmed as "pressure-supported" systems.

The data reveal a host of individual peculiarities, like: wiggles, strong gradients, and asymmetries in the rotation curve and/or in the velocity dispersion profile, thus showing that the presence of kinematically distinct components and/or triaxiality is a common characteristic of this class of object.
\end{abstract}

Key words: galaxies: kinematics and dynamics galaxies: elliptical and lenticular, $\mathrm{cD}-$ galaxies: clusters: individual: Fornax — galaxies: structure galaxies: fundamental parameters

Send offprint requests to: A.W. Graham, ali@mso.anu.edu.au

* Based on observations collected at Siding Spring Observatory.

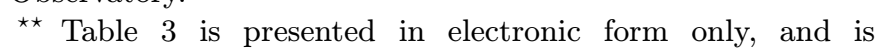
available from the CDS, Strasbourg via anonymous ftp to cdsarc.u-strasbg.fr (130.79.128.5) or via http://cdsweb.ustrasbg.fr/Abstract.html

\section{Introduction}

The scaling relations between some global properties of ellipticals (Es), known as the "Fundamental Plane" (FP; Dressler et al. 1987; Djorgovski \& Davis 1987), is clearly a manifestation of a great "regularity" in the properties of Es. Although a proof of its "universality" is still lacking, the FP has been widely applied to various problems: from the study of peculiar motions (Lynden-Bell et al. 1988; Jørgensen et al. 1996), to the study of the evolution of M/L ratios (van Dokkum \& Franx 1996; Kelson et al. 1997), and measurements of $q_{0}$ (Bender et al. 1998).

The "regularity" of the E's properties, shown by the FP, must be connected to their gross structural characteristics and to their formation and evolutionary mechanisms (Djorgovski et al. 1995 and references therein). To understand this connection is however a very hard task, especially in view of the problems posed by the "thinness" of the FP itself (Ciotti et al. 1996). Apart from the obvious similarities, it is a matter of fact that Es do not constitute a homogeneous family of galaxies: Es not only have substantially different photometric and kinematical properties, but they also host a variety of subcomponents, like inner disks, counter-rotating cores, outer rings, dust lanes, etc. (Illingworth 1983; Kormendy \& Djorgovski 1989; Caon et al. 1993; Michard \& Marchal 1994; Capaccioli \& Longo 1994; Scorza \& Bender 1996; Graham et al. 1996; Graham \& Colless 1997; Busarello et al. 1997; Prugniel \& Simien 1997; Carollo et al. 1997, and references therein).

The possibility that many Es have disks that escape photometric detection (Simien \& de Vaucouleurs 1986; Capaccioli 1987; Rix \& White 1990; Saglia et al. 1997), and the dichotomy between the two families of boxy and disky Es lead to the possibility of a new classification scheme, in which galaxies formerly belonging to the same Hubble type are separated according to isophotal shape, and in which a continuous sequence exists between Es and S0s (Kormendy \& Bender 1996). Since the above scenario is manifestly at odds with the "regularity" of the FP, the question arises to what extent the "global" quantities 
entering into the construction of the $\mathrm{FP}$ are representative of the real status of the galaxies.

In this respect, an important question is whether the central velocity dispersion $\sigma_{0}$ is fully representative of the dynamical status of a galaxy. There is much evidence that this is not the case: due to differences in velocity dispersion profiles and anisotropy, masses derived from $\sigma_{0}$ can be wrong by a factor of ten (Tonry 1983; Richstone \& Tremaine 1986; Mathews 1988); in presence of central, kinematically distinct sub-components, the value of $\sigma_{0}$ is likely to be more connected to the sub-component than to the galaxy as a whole; Es are dynamically non-homologous systems: the slope of the velocity dispersion profile is correlated to $\sigma_{0}$ in such a way that there is a systematic departure of the kinetic energy from a simple scaling of $\sigma_{0}$ (Graham \& Colless 1997; Busarello et al. 1997); finally, the rotation, although not dominant in Es, plays a nonnegligible role (Busarello et al. 1992; Prugniel \& Simien 1994, 1996; Busarello et al. 1997; D’Onofrio et al. 1997).

To fully understand the complex kinematics of Es, and to analyze the connection between the individual properties of Es and the scaling laws, it is essential to have access to kinematical data as accurate, extended and homogeneous as possible. One of the objectives of the present work is to obtain homogeneous sets of data for magnitudelimited samples of elliptical galaxies belonging to different clusters.

In the present paper we present new stellar kinematical data for 13 galaxies in the Fornax cluster.

Section 2 describes the observations and the data reduction. In Sect. 3, we give a short description of the major features of each of the kinematical profiles. Section 4 provides a comparison of our data with previous observations, and in Sect. 5 we briefly summarize our findings. The data themselves are presented in Appendix A and in Table 3 (available in electronic form from the CDS).

\section{Observations and reduction}

\subsection{Observations}

Ferguson (1989) lists 340 likely Fornax cluster members within an area of $\sim 40 \mathrm{sq}$ deg centered on the cluster. Of these, 14 are classified as elliptical galaxies brighter than $B_{\mathrm{T}}=15.0$ mag. We were able to obtain kinematical data for 12 of these galaxies making our investigation $86 \%$ complete at that limiting magnitude. In addition, we have obtained kinematical data for FCC 119, (classified as a S0 galaxy by Ferguson, and for which we give only the central velocity dispersion (CVD) and $V_{\max }$ ).

The spectra were obtained over two runs in November and December 1996 using the blue arm of the Double Beam Spectrograph attached to the Australian National University's $2.3 \mathrm{~m}$ telescope at Siding Spring Observatory. Dichroic \#3 was used, providing greater than $95 \%$ transmission at all wavelengths between $4000-6000 \AA$.
A $1200 l \mathrm{~mm}^{-1}$ grating was used, with a dispersion of $0.555 \AA$ pixel $^{-1}$ over a range of $1000 \AA$ centered on $5200 \AA$. The CCD used was a SITe chip $(1752 \times 532)$ with $15 \mu \mathrm{m}$ pixels. The spatial scale on the chip was $0.91^{\prime \prime}$ pixel $^{-1}$. We used a spectrograph slit of $2^{\prime \prime}$ on the sky and of length greater than the spatial extent of the CCD. The best collimator focus gave a FWHM for the arc lines of 2.7 pixels or $1.50 \AA$, giving a resolution of $86 \mathrm{~km} \mathrm{~s}^{-1}$ at $5200 \AA$. During the observations the seeing varied from 1.5 to 2.5 arcsec.

The observed galaxies are listed in Table 1, along with their photometric parameters (taken from Caon et al. 1994, we also refer to this paper for a detailed photometric description). We obtained our estimates of the galaxy major-axis PAs based on careful inspection of the position angle (PA) profiles (Caon et al. 1994). Only our estimate for the major-axis PA of NGC 1419 differed from the value adopted in Caon et al. (1994), where we have adopted 50 rather than $65^{\circ}$. The number of spectra for each galaxy and the total exposure times used are also listed in the table. The spectra were exposed in $30-45$ min blocks. A Ne-Ar lamp was observed before and after each exposure for wavelength calibration and four template stars (of spectral types from G8III to K3III) were observed at the beginning, middle, and end of each night. The usual dome and sky flats were taken, as were bias frames and measurements of the dark current.

\subsection{Data reduction}

The data reduction included, besides the usual CCD cosmetics, noise removal along the spatial direction by adaptive filtering techniques (Richter et al. 1992). After wavelength calibration and sky subtraction, the spectra were normalized to the continuum, obtained by fitting with a 6 th order polynomial. The reliability of the sky subtraction was iteratively tested by comparing the brightness profiles of the sky-subtracted spectra to the profiles from Caon et al. (1994). The slit function, as derived from twilight spectra, varied by at most $0.5 \%$, so that no further correction was needed. The low-frequency residual variations were reduced by filtering in Fourier space. Finally, the spectra were processed by the Fourier Correlation Quotient (FCQ) technique (Bender 1990). All four template stars were used, and the relative results were compared to test their consistency: as expected, no relevant difference was detected (see Bender 1990). In order to test the reliability of the observed features, we compared the velocity dispersion profiles (VDPs) and the rotation curves (RCs) obtained by adding the different spectra of each individual galaxy before and after the final stage of processing (the FCQ), obtaining fully consistent results. As a further test, we also reduced the data without filtering and, although the data were obviously more noisy in the fainter regions, the observed features were still present. 
Table 1. Galaxy sample and photometric parameters

\begin{tabular}{|c|c|c|c|c|c|c|c|c|c|}
\hline $\begin{array}{l}\alpha_{1950} \\
(\mathrm{~h} \mathrm{~m} \mathrm{~s})\end{array}$ & $\begin{array}{l}\delta_{1950} \\
(\circ / / \prime)\end{array}$ & NGC \# & FCC Type & $\begin{array}{c}B_{\mathrm{T}} \\
(\mathrm{mag})\end{array}$ & $\begin{array}{r}r_{\mathrm{e}} \\
\left({ }^{\prime \prime}\right)\end{array}$ & $\begin{array}{c}\mu_{\mathrm{e}} \\
(\mathrm{mag} / \mathrm{sq} \operatorname{arcsec})\end{array}$ & $\begin{array}{r}\mathrm{PA} \\
\circ\end{array}$ & $\begin{array}{c}\# \\
\text { spec. }\end{array}$ & $\begin{array}{l}\text { Exp. } \\
\text { (hr) }\end{array}$ \\
\hline 32435.9 & -355310 & NGC 1336 & $47 \mathrm{E} 4$ & 13.3 & 30 & 23.6 & 20 & 2 & 1 \\
\hline $\begin{array}{lll}326 & 06.1\end{array}$ & -322726 & NGC 1339 & $63 \mathrm{E} 4$ & 12.7 & 15 & 21.5 & 175 & 4 & 2 \\
\hline 33135.6 & -334419 & & $119 \mathrm{~S} 0$ & 15.1 & 19 & 24.4 & 40 & 3 & 2.25 \\
\hline 33234.1 & -354239 & & $136 \mathrm{dE} 2$ & 14.7 & 26 & 24.7 & 170 & 5 & 2 \\
\hline 33303.3 & -352006 & NGC 1373 & 143 E3 & 14.2 & 11 & 22.4 & 140 & 7 & 3.5 \\
\hline 33321.1 & -352329 & NGC 1374 & $147 \mathrm{E} 0$ & 11.9 & 26 & 22.3 & 120 & 2 & 1 \\
\hline 33408.7 & -353622 & NGC 1379 & $161 \mathrm{E} 0$ & 12.0 & 24 & 22.0 & 07 & 2 & 0.75 \\
\hline 33634.2 & -353646 & NGC 1399 & 213 E0 & 10.0 & 134 & 24.2 & 112 & 5 & 3 \\
\hline 33657.3 & -354517 & NGC 1404 & $219 \mathrm{E} 2$ & 10.9 & 26 & 21.2 & 160 & 3 & 2.5 \\
\hline 33850.3 & -374009 & NGC 1419 & 249 E0 & 13.6 & 9 & 21.7 & 50 & 2 & 1 \\
\hline 34024.6 & -353306 & NGC 1427 & $276 \mathrm{E} 4$ & 11.8 & 39 & 22.8 & 77 & 2 & 1 \\
\hline 34027.5 & -351844 & NGC 1428 & 277 E5 & 13.8 & 12 & 21.9 & 115 & 7 & 3.25 \\
\hline 34843.8 & -360329 & & $335 \mathrm{E}$ & 14.4 & 19 & 23.5 & 45 & 4 & 2.25 \\
\hline
\end{tabular}

The columns respectively list the R.A. and Dec. of each galaxy, the NGC number (where available) and Fornax Cluster Catalogue number (FCC; Ferguson 1989), morphological type according to Ferguson (1989), total apparent blue magnitude, effective halflight radius, and associated blue surface brightness at that radius and major-axis position angle (measured E from N). The number of spectra and the total exposure time for each object are also given. The photometric parameters are taken from Caon et al. (1994).

A lower limit of $\sim 35 \mathrm{~km} \mathrm{~s}^{-1}$ in the measurable velocity dispersion, due to the instrumental setup, was also verified on the template stars. The uncertainties in the data are those derived by the FCQ procedures, basically arising from the fit of the broadening function, and are explained in Bender (1990).

\section{Results}

The figures in Appendix A and Table 3 (available in electronic form) present the RCs and VDPs. The major-axis $\mathrm{PA}$ points from left to right in the figures, that is, the right hand side in the plots corresponds to the easterly half of the galaxies major-axis. In addition, in Table 2 we list the values of the CVD and of the maximum observed rotation velocity $V_{\max }$.

The major kinematical features of each galaxy are outlined in the following. The term "kinematically distinct" is used in the present section in a descriptive sense only, without attaching to it any particular interpretation (see Sect. 5). By "inner (or central) $x^{\prime \prime}$ ", we refer to a radius (not a diameter).

\section{NGC 1336}

The VDP dips $\sim 5 \mathrm{~km} \mathrm{~s}^{-1}$ over the central $2^{\prime \prime}$ and contains additional oscillations at larger radii, having amplitudes of $10 \mathrm{~km} \mathrm{~s}^{-1}$. Smoothing these out, the VDP shows negligible signs of decreasing until a radius of $18^{\prime \prime}$, remaining roughly flat at $\sim 100 \mathrm{~km} \mathrm{~s}^{-1}$. The RC is symmetric, being approximately flat over the inner $\sim 5^{\prime \prime}$, where a strong isophotal twist is known to take place (up to $60^{\circ}$; Caon et al. 1994). Beyond this radius, the RC displays a wave-like appear- ance about a zero mean rotational velocity, with an amplitude of $20 \mathrm{~km} \mathrm{~s}^{-1}$. Such kinematics is indicative of the presence of a bar (Sparke \& Sellwood 1987; Bettoni 1989, and references therein). Concerning this point, it is also worth noting that the normalized Fourier coefficient $a_{4}$ is high over the inner 10" (Caon et al. 1994).

\section{NGC 1339}

The $\mathrm{RC}$ is well traced out to $2 R_{\mathrm{e}}$, having a steep gradient of $200 \mathrm{~km} \mathrm{~s}^{-1} \mathrm{kpc}^{-1}$ (assuming a distance of $18 \mathrm{Mpc}$ ) over the inner $5^{\prime \prime}$ radius. The $\mathrm{E}$ and $\mathrm{W}$ side of the $\mathrm{RC}$ largely flatten out after $10^{\prime \prime}$. The VDP decreases linearly from $175 \mathrm{~km} \mathrm{~s}^{-1}$ to $100 \mathrm{~km} \mathrm{~s}^{-1}$ at $10^{\prime \prime}$ on both sides. After $20^{\prime \prime}$, both sides of the VDP increase some $50 \mathrm{~km} \mathrm{~s}^{-1}$, reaching the outer data point $\left(30^{\prime \prime}\right)$.

\section{FCC 119}

Catalogued as a S0 galaxy by Ferguson (1989), we only provide the central velocity dispersion $\sigma_{0}=51 \pm$ $10 \mathrm{~km} \mathrm{~s}^{-1}$ and maximum observed rotational velocity $V_{\max }=27 \pm 19 \mathrm{~km} \mathrm{~s}^{-1}$ for this galaxy, the faintest of our sample.

\section{FCC 136}

This faint galaxy is classified as dE2 by Ferguson (1989) and as SAB0 in RC3. The VDP is poorly defined around $40 \mathrm{~km} \mathrm{~s}^{-1}$, close to our measurement limit. There is marginal evidence for rotation within the wavy RC, which increases to $15 \mathrm{~km} \mathrm{~s}^{-1}$ by the outer data points $\left(15^{\prime \prime}\right)$. The shape of the $\mathrm{RC}$, together with the fact that the inner isophotes $\left(<4^{\prime \prime}\right)$ twist through $30^{\circ}$, while the normalized Fourier coefficient $a_{4}$ is high (Caon et al. 1994) supports the RC3 classification as a barred galaxy. 


\section{NGC 1373}

The symmetric RC reveals a small degree of rotation, peaking at $15 \mathrm{~km} \mathrm{~s}^{-1}$. The inner $10-15^{\prime \prime}$ of the VDP has a wave-like appearance, with a $5 \mathrm{~km} \mathrm{~s}^{-1}$ wave amplitude producing no obvious, and several local, maxima around $80 \mathrm{~km} \mathrm{~s}^{-1}$. The VDP is asymmetric: the E side is flat out to $14^{\prime \prime}$ from the center and then decreases to $\sim 30 \mathrm{~km} \mathrm{~s}^{-1}$ at $20^{\prime \prime}$, while the $\mathrm{W}$ side slowly decreases starting from $6^{\prime \prime}$, to reach a minimum of $40 \mathrm{~km} \mathrm{~s}^{-1}$ at $24^{\prime \prime}$.

The overall shape of the VDP and the presence of strong isophotal twisting (Caon et al. 1994) are, in this case, also indicative of the presence of a bar.

\section{NGC 1374}

The inner gradient of the rotation velocity is very steep $\left(250 \mathrm{~km} \mathrm{~s}^{-1} \mathrm{kpc}^{-1}\right)$. After reaching a first maximum at $2^{\prime \prime}$ from the center, the $\mathrm{RC}$ remains approximately flat on both sides, until reaching a second maximum at $30-35^{\prime \prime}$. The asymmetries of the RC correspond to the analogous behavior of the VDP which, after an initial strong gradient, and after reaching a plateau of $145 \mathrm{~km} \mathrm{~s}^{-1}$ at $7-10^{\prime \prime}$, shows different slopes on the two sides. The VDP is approximately flat, at $120 \mathrm{~km} \mathrm{~s}^{-1}$, on the $\mathrm{E}$ side in the range $15-35^{\prime \prime}$ before dropping to a minimum of $70 \mathrm{~km} \mathrm{~s}^{-1}$ at the same position $\left(35^{\prime \prime}\right)$ of the maximum of the RC. However, the W side of the VDP shows evidence for increasing over the radial range corresponding to the second rise in the $\mathrm{W}$ side of the RC.

\section{NGC 1379}

The kinematics of this galaxy, although rather symmetric in its overall appearance, presents a number of remarkable small-scale asymmetries. The series of minima and maxima in both the RC and the VDP are clearly anticorrelated, the maxima of the VDP corresponding to minima in the $\mathrm{RC}$ and vice-versa. There is evidence for a small $\left(5^{\prime \prime}\right)$ inner disk, coming from the steepest part of the RC, clearly connected to the inflexion in both sides of the VDP at this radius. There is also marginal evidence for a central distinct component corresponding to the inflexion of the RC.

\section{NGC 1399}

This is the largest Fornax galaxy, at the center of the cluster. Its dominant status is confirmed by its large velocity dispersion (notice however that the peak value of $\sigma$ is underestimated by our data because of the relatively poor seeing, see Sect. 4). The VDP quickly declines to $250 \mathrm{~km} \mathrm{~s}^{-1}$ by a radius of $10^{\prime \prime}$, to remain approximately flat to the outer data points. The RC presents strong evidence for a kinematically distinct inner component, with an inflexion point evident over the central $4^{\prime \prime}$. The $\mathrm{W}$ side of the $\mathrm{RC}$ reaches a maximum value of $30 \mathrm{~km} \mathrm{~s}^{-1}$ at $15^{\prime \prime}$, where it then steadily falls to zero by $55^{\prime \prime}$. It then changes sign, increases to $10 \mathrm{~km} \mathrm{~s}^{-1}$ at $60^{\prime \prime}$ and then decreases again. The wiggles of the $\mathrm{RC}$ in the range $40-70^{\prime \prime}$ are associated to wiggles in the VDP in the same range and with the same amplitude. The E side of the $\mathrm{RC}$ appears identical to the $\mathrm{W}$ side until reaching $30^{\prime \prime}$ where it increases to $50 \mathrm{~km} \mathrm{~s}^{-1}$ to then stagger back down to a rotational speed of $20 \mathrm{~km} \mathrm{~s}^{-1}$ at the outer data point. The E side of the VDP resembles the $\mathrm{W}$ side until at $55^{\prime \prime}$ where it decreases by $40 \mathrm{~km} \mathrm{~s}^{-1}$ over the following $5^{\prime \prime}$ and then starts climbing, still increasing at the outer data point.

\section{NGC 1404}

The RC has a steep gradient of $200 \mathrm{~km} \mathrm{~s}^{-1} \mathrm{kpc}^{-1}$ inside $10^{\prime \prime}$, with the exception of the two inner arcseconds, where the RC remarkably flattens. At $r \sim 10^{\prime \prime}$ and $r \sim 22^{\prime \prime}$ there are two local maxima around $V \sim 100 \mathrm{~km} \mathrm{~s}^{-1}$, which are symmetric with respect to the center. The rotation velocity starts increasing again beyond $r=30^{\prime \prime}$, reaching $\sim 140 \mathrm{~km} \mathrm{~s}^{-1}$ at the last observed point: the RC seems actually to be increasing even beyond 3 effective radii.

In the central region the VDP dips by $15 \mathrm{~km} \mathrm{~s}^{-1}$ from the two local maxima situated at $\pm 2^{\prime \prime}$ from the center. The velocity dispersion then decreases at a nearly constant rate in the inner $20^{\prime \prime}$, until reaching a plateau around $200 \mathrm{~km} \mathrm{~s}^{-1}$. In the range from $40^{\prime \prime}$ to $50^{\prime \prime}$ the VDP decreases before increasing to a local maximum of $180 \mathrm{~km} \mathrm{~s}^{-1}$ around 1 arcmin from the center.

\section{NGC 1419}

This galaxy has a very similar RC to NGC 1336, with $\sim 15 \mathrm{~km} \mathrm{~s}^{-1}$ wiggles observed out to $2 R_{\mathrm{e}}$. The VDP is largely flat (at $\sim 120 \mathrm{~km} \mathrm{~s}^{-1}$ ) over the inner $9^{\prime \prime}\left(=1 R_{\mathrm{e}}\right.$ ), then it steadily decreases to $\sim 70 \mathrm{~km} \mathrm{~s}^{-1}$ reaching the outer radius limit of the data. Like NGC 1336, this galaxy also has a very strong isophotal twist $\left(\sim 100^{\circ}\right)$ over the inner region, where the $\mathrm{RC}$ is observed to remain flat. Also in this case we thus find evidence for a possible bar-like structure.

\section{NGC 1427}

This galaxy has a wiggly VDP which peaks around $180 \mathrm{~km} \mathrm{~s}^{-1}$ and falls to $140 \mathrm{~km} \mathrm{~s}^{-1}$ at the data boundary. The CVD is $\sim 13 \mathrm{~km} \mathrm{~s}^{-1}$ below the peak value which occurs $4^{\prime \prime} \mathrm{W}$ from the center. The RC, after reaching a first maximum of $\sim 25 \mathrm{~km} \mathrm{~s}^{-1}$ at $5^{\prime \prime}$, shows a series of secondary maxima on both sides, that are anti-correlated to the wiggles in the VDP. There is also marginal evidence for an inflexion in the center which, if real, and if related to the dip in the VDP could constitute kinematical evidence for the central faint disk detected by Carollo et al. (1997, see also Forbes et al. 1995).

\section{NGC 1428}

The presence of a star superimposed on the nucleus of this galaxy prevents one from obtaining kinematical data in the central $4^{\prime \prime}$. In the observable region, the rotation velocity steadily increases, reaching $\sim 70 \mathrm{~km} \mathrm{~s}^{-1}$ at $2.7 a_{\mathrm{e}}$ on both sides. Small amplitude waves are present around $15^{\prime \prime}$ from the center in both the RC and the VDP. A general, though weak, asymmetry is observed in both profiles: on 
the $\mathrm{E}$ side the velocity dispersion is lower and the rotation velocity is higher than on the $\mathrm{W}$ side. The increase of the VDP beyond $20^{\prime \prime}$ in the E side is, on the other hand, not real, as can be inferred from the large uncertainties.

\section{FCC 335}

This is a faint galaxy, with a roughly flat VDP of $40 \mathrm{~km} \mathrm{~s}^{-1}$, close to the lower limit of our measurements. There is marginal evidence for an increase on the $\mathrm{W}$ side of the VDP of $\sim 10 \mathrm{~km} \mathrm{~s}^{-1}$ around $13^{\prime \prime}$. This is associated with a decrease of the RC by $15 \mathrm{~km} \mathrm{~s}^{-1}$ over the same interval. The $\mathrm{RC}$ itself peaks at a maximum rotational velocity of $\sim 25 \mathrm{~km} \mathrm{~s}^{-1}$ at $10^{\prime \prime}=0.5 R_{\mathrm{e}}$, where the $\mathrm{W}$ side then decreases its rotation and the $\mathrm{E}$ side remains flat to the boundary of our data.

\section{Comparison with previous data}

Out of the 13 galaxies in the present sample, five (FCC 119, FCC 136, NGC 1373, NGC 1428 and FCC 335) have no previously published kinematic data, while the others have been studied by different groups. Figures 1 and 2 in Appendix B show our data compared to the previous studies. We summarize here how our data compare with the other groups results.

Three of our sample galaxies are in common with the study by Franx et al. (1989; hereafter FIH). Our RC of NGC 1379 is in close agreement, while our VDP has a shallower gradient compared to that by FIH, but agrees with the CVD measure of $\sim 135 \mathrm{~km} \mathrm{~s}^{-1}$. It is interesting to note that the minor-axis VDP of FIH is instead fully consistent both with ours and with the VDP of D'Onofrio et al. (1995). Both the VDP and the RC of NGC 1399 and NGC 1404 agree with those by FIH. Bicknell et al. (1989) and Stiavelli et al. (1993) presented kinematical profiles of NGC 1399 at $\mathrm{PA}=84^{\circ}$. Our VDP is consistent with their data, while the comparison with the RC by Bicknell et al. (1989) is made difficult by the larger uncertainties in their results (their $\mathrm{RC}$ is actually consistent with any $\mathrm{RC}$ not exceeding $30 \mathrm{~km} \mathrm{~s}^{-1}$ ). Notice, however, that there is a difference of $28^{\circ}$ in PA. We are also consistent with the kinematical profiles by Van der Marel \& Franx (1993) for NGC 1374, the only galaxy from their sample in common with ours.

The study with the largest number (8) of galaxies in common with us, is that by D'Onofrio et al. (1995; hereafter D95, see also Longo et al. 1994). Their kinematical profiles are folded around the photometric centers. In general, the agreement between the two sets of data is good, with only some of the discrepancies in the profiles worth mentioning (the CVDs will be addressed below). The first discrepancy is the up-turn in the VDP of NGC 1339, apparent in the data of D95 at $4-6$ arcsec. This difference cannot be attributed to the folding process used by D95 and it is at odds with our data which give no indication of its presence. Another discrepancy is the hump in the RC of NGC 1427 over the inner 6 arcsec. D95 show it to reach a maximum rotational speed of $\sim 45 \mathrm{~km} \mathrm{~s}^{-1}$, whereas our data suggests it does not reach any more than $\sim 25-30 \mathrm{~km} \mathrm{~s}^{-1}$. The data for NGC 1404 are in agreement in the inner $10^{\prime \prime}$, even if the central dip in the VDP is less pronounced in the D95 data. The comparison is difficult beyond that radius, due to the heavy smoothing of the D95 data, that eventually lead to a wrong negative gradient of the RC. Finally, the RC of NGC 1339 in D95 is steeper than ours in the inner $5^{\prime \prime}$, while the opposite happens for NGC 1374, where D95 fail do detect the maximum at $3^{\prime \prime}$ from the center.

The comparison of the CVDs needs some prior comments. Generally, our CVD measurements agree with the values given in the above-mentioned works, and with those listed by McElroy (1995), with the exception of NGC 1399, for which McElroy gives a value of $308 \mathrm{~km} \mathrm{~s}^{-1}$. D95 give $420 \pm 27 \mathrm{~km} \mathrm{~s}^{-1}$, that is still under the $430 \mathrm{~km} \mathrm{~s}^{-1}$ derived by Stiavelli et al. (1993) using core resolution techniques applied to observations performed under $0.7^{\prime \prime}$ seeing. Our CVDs are systematically lower than those by D95, that were obtained under better seeing conditions (private communication). On the other hand, since the smoothing effect of the seeing is stronger for the steeper VDPs, and since the gradient of the VDP is correlated to the CVD (Busarello et al. 1997), we expect the lower CVDs to be less affected by the smoothing. The two sets of data are related by: $\log \left(\sigma_{0}^{D 95}\right)=1.10( \pm .04) \times$ $\log \left(\sigma_{0}^{\text {this work }}\right)-0.17( \pm .1)$ (see Fig. 1$)$. We can then use this relation to estimate the "corrections" to the CVDs for the other objects of our sample. These "corrected" CVDs are also listed in Table 2.

\section{Conclusions}

Of the 12 ellipticals studied here, five (NGC 1339, NGC 1374, NGC 1379, NGC 1404 and NGC 1419) are rotationally supported systems, having positive rotation parameter $\left(\log \left(\frac{V}{\sigma}\right)^{*}>0\right)$. The interest in this result is strengthened by the fact that four of these galaxies are E0/E1 systems, the only remaining round system in the sample being NGC 1399. NGC 1374, NGC 1379 and NGC 1404 are most probably S0 galaxies (due to the overall constancy of their RCs; see also Fisher 1997, and the case of NGC 3379 presented in Statler et al. 1997), although a more complex structure has to be invoked to explain their kinematical features. The RCs of NGC 1374 and NGC 1404, in particular, may reveal the presence of a double-disk structure (cf. Seifert \& Scorza 1996). The rotation parameter of NGC 1339 is consistent with that of an isotropic oblate model, but the shape and the asymmetry of both the VDP and the RC indicate a significant departure from a "simple" isotropic rotator.

Four objects (NGC 1336, FCC 136, NGC 1373, and NGC 1419) are most probably SB0 galaxies: NGC 1336 
Table 2. Summary of the kinematical properties

\begin{tabular}{lrrrrrrr}
\hline Ident. & $\begin{array}{r}V_{\text {hel }} \\
\mathrm{km} \mathrm{s}^{-1}\end{array}$ & $\begin{array}{r}\sigma_{0} \\
\mathrm{~km} \mathrm{~s}^{-1}\end{array}$ & $\begin{array}{r}V_{\max } \\
\mathrm{km} \mathrm{s}^{-1}\end{array}$ & $r_{\max }$ & $\frac{r_{\max }}{a_{\mathrm{e}}}$ & $\sigma_{0}(\mathrm{D} 95)$ & $\log \left(\frac{V}{\sigma}\right)^{*}$ \\
$\mathrm{~km} \mathrm{~s}^{-1}$ & \\
\hline NGC 1336 & $1418 \pm 3$ & $108 \pm 4$ & $17 \pm 10$ & 25 & 0.8 & $115 \pm 6$ & -0.57 \\
NGC 1339 & $1392 \pm 3$ & $174 \pm 6$ & $134 \pm 8$ & 30 & 2.0 & $190 \pm 6$ & 0.08 \\
FCC 119 & $1374 \pm 7$ & $51 \pm 10$ & $27 \pm 9$ & $\ldots \ldots$ & $\ldots \ldots$ & 50 & 0.12 \\
FCC 136 & $1205 \pm 1$ & $39 \pm 2$ & $16 \pm 5$ & 15 & 0.6 & 37 & -0.11 \\
NGC 1373 & $1334 \pm 2$ & $79 \pm 1$ & $14 \pm 7$ & 24 & 2.2 & 80 & -0.49 \\
NGC 1374 & $1294 \pm 2$ & $187 \pm 5$ & $96 \pm 9$ & 40 & 1.5 & $225 \pm 10$ & 0.22 \\
NGC 1379 & $1324 \pm 2$ & $135 \pm 4$ & $30 \pm 5$ & 60 & 2.5 & $140 \pm 10$ & 0.11 \\
NGC 1399 & $1425 \pm 4$ & $353 \pm 19$ & $51 \pm 5$ & 70 & 0.5 & $420 \pm 27$ & -0.36 \\
NGC 1404 & $1947 \pm 4$ & $241 \pm 11$ & $140 \pm 12$ & 80 & 3.1 & $260 \pm 11$ & 0.22 \\
NGC 1419 & $2133 \pm 2$ & $118 \pm 5$ & $14 \pm 8$ & 18 & 2.0 & $125 \pm 8$ & $(\infty)$ \\
NGC 1427 & $1388 \pm 3$ & $170 \pm 7$ & $27 \pm 2$ & 40 & 1.0 & $180 \pm 5$ & -0.62 \\
NGC 1428 & $1640 \pm 8$ & $\ldots \ldots$. & $83 \pm 8$ & 33 & 2.7 & $\ldots \ldots$. & $\ldots \ldots$. \\
FCC 335 & $1430 \pm 2$ & $43 \pm 3$ & $24 \pm 4$ & 15 & 0.8 & 41 & -0.14 \\
\hline
\end{tabular}

The following quantities are listed for each galaxy: heliocentric velocity (Col. 2); central velocity dispersion (Col. 3); maximum observed rotation velocity (Col. 4); radial range of the kinematical data in arcseconds and in units of $a_{\mathrm{e}}$ (Cols. 5 and 6 respectively), except for the S0 galaxy FCC 199 (see text); central velocity dispersion from D95 and the "corrected" value (those without error, see text). The last column gives the "rotation parameter" $\log \left(\frac{V}{\sigma}\right)^{*}$ which measures the relevance of the rotational support (notice that this value is virtually infinite for NGC 1419, for which $\epsilon_{\mathrm{e}}=0.00$ (Caon et al. 1994)). For NGC $1428, V_{\text {hel }}$ has been derived by interpolation of the RC in the central region. For FCC $119 V_{\max }$ is an upper limit.

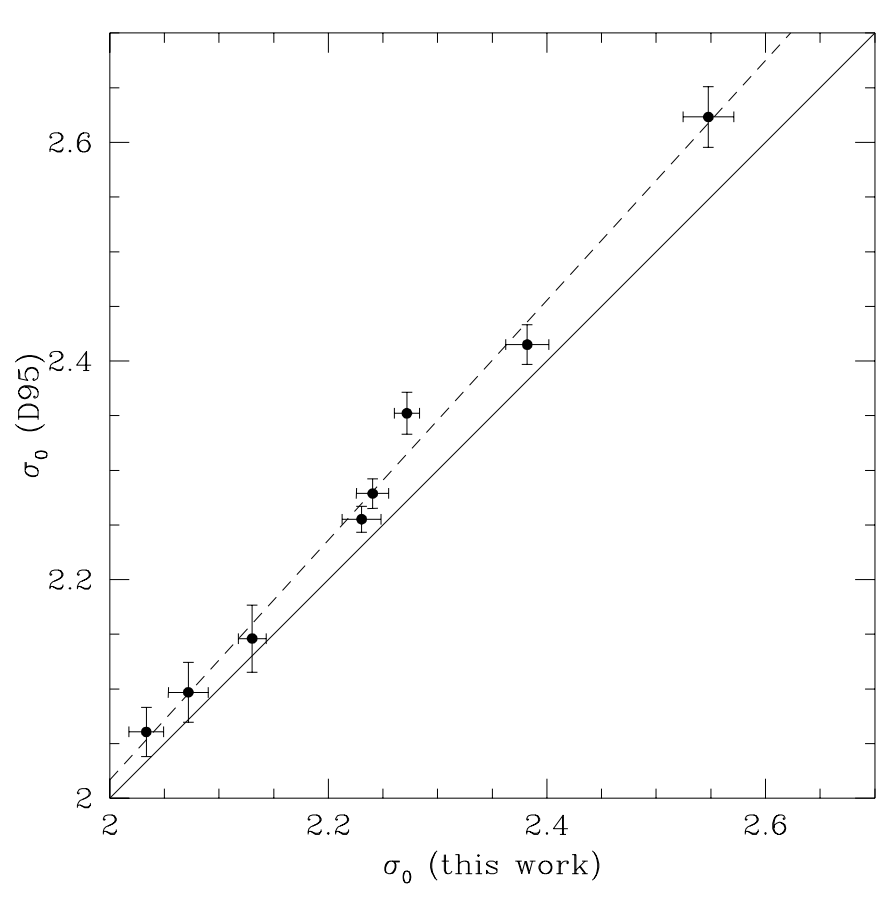

Fig. 1. Comparison of the CVDs in this work to those by D'Onofrio et al. (1995). The dashed line is the least square fits between the two samples $\left(\log \left(\sigma_{0}^{\mathrm{D} 95}\right)=1.10( \pm .04) \times\right.$ $\left.\log \left(\sigma_{0}^{\text {this work }}\right)-0.17( \pm .1)\right)$, while the continuous line represents the equality between the two quantities. The difference between the two sets is accounted for by the difference in the seeing conditions and NGC 1419, in particular, have kinematical profiles identical to the SB0 galaxy IC 456 (Bettoni 1989, see also Galletta 1996). This conclusion is also strongly supported by the photometric properties, all these galaxies having strong isophotal twisting (see the photometric profiles in Caon et al. 1994).

The three remaining galaxies are definitely "dynamically hot" systems. FCC 335 is a faint galaxy $\left(M_{B}=\right.$ -16.8 for $H_{0}=75 \mathrm{~km} \mathrm{~s}^{-1} \mathrm{Mpc}^{-1}$ ) with low surface brightness $\left(\mu_{\mathrm{e}}=23.5 \mathrm{~B} \mathrm{mag} / \operatorname{arcsec}^{2}\right)$, low velocity dispersion $\left(\sigma_{0}=43 \mathrm{~km} \mathrm{~s}^{-1}\right)$ and low rotation $\left(V_{\max }=24 \mathrm{~km} \mathrm{~s}^{-1}\right)$. NGC 1399 and NGC 1427 are instead bright galaxies, with high (and roughly constant) velocity dispersions, and relatively slow rotation: this is exactly what one expects from "true" giant ellipticals. For both objects the present data show complex kinematics: the already-known kinematically distinct core in NGC 1399 plus a second clear kinematically distinct component visible at $10^{\prime \prime}$ from the center. Both from the VDP and from the RC of NGC 1427 there is evidence for kinematically distinct components at the inner $2^{\prime \prime}$ and inside $10^{\prime \prime}$ from the center; NGC 1427 also shows anti-correlated wiggles in both the $\mathrm{RC}$ and the VDP.

In summary: it seems that more than half of the galaxies in the present sample are, at least from the kinematical point of view, misclassified S(A-B)0s. Morphological classification by visual inspection of images, apparently results in a dramatic over-estimate of the true number of "dynamically hot" stellar systems. The catalog of Ferguson (1989) lists 58 Fornax galaxies with $B_{\mathrm{T}}<15.0 \mathrm{mag}$, from the 14 listed as having morphological type E, only 3 turn out to 
be true elliptical galaxies in the classical sense of the word; indicating that these objects are in fact quite rare. Most of the objects, moreover, show complex kinematical profiles, like kinematically distinct components, wiggles, and asymmetries.

The presence of (dynamically decoupled) sub-components is one of the possible explanations of such complex kinematics. Complicated velocity fields may arise as well from triaxial systems due to competing contributions of different families of orbits (Statler 1991a,b; Statler et al. 1997, and references therein), like, for instance, the possible presence of a substantial fraction of retrograde orbits (Wozniak \& Pfenninger 1997; compare their Fig. 5c to some of our RCs).

A concluding remark is in order: all the data presented here show very complicated kinematics, which is only in part accounted for by misclassification of S0s into Es. It seems, instead, conceivable that complex kinematics is a common, and dynamically stable, characteristic of elliptical galaxies.

Acknowledgements. We are grateful to F. Bertola, D. Burstein, M. Capaccioli, K. Freeman, Ph. Prugniel, F. Simien, and L. Sparke for helpful discussions and suggestions. We are also grateful to the staff of MSSSO and to K. McKenzie for her telescope assistance. We thank the anonymous referee whose comments helped us express some points more clearly.

\section{References}

Bender R., 1990, A\&A 229, 441

Bender R., Saglia R.P., Ziegler B., et al., 1998, ApJ 493, 529

Bettoni D., 1989, AJ 97, 79

Bicknell G.V., Carter D., Killeen N.E.B., Bruce T.E.G., 1989, ApJ 336, 639

Busarello G., Longo G., Feoli A., 1992, A\&A 262, 52

Busarello G., Capaccioli M., Capozziello S., Longo G., Puddu E., 1997, A\&A 320, 415

Caon N., Capaccioli M., D'Onofrio M., 1993, MNRAS 265, 1013

Caon N., Capaccioli M., D’Onofrio M., 1994, A\&AS 106, 199

Capaccioli M., 1987, IAU Symp. 127, T. de Zeeuw (ed.). Kluwer: Dordrecht, p. 47

Capaccioli M., Longo G., 1994, A\&AR 5, 293

Carollo C.A., Franx M., Illingworth G.D., Forbes D., 1997, ApJ 481,710

Ciotti L., Lanzoni B., Renzini A., 1996, MNRAS 282, 1

De Vaucouleurs G., de Vaucouleurs A., Corwin H.G., et al., 1991, Third Reference Catalogue of Bright Galaxies. Springer, N.Y., RC3

Djorgovski G., Davis M., 1987, ApJ 313, 59

Djorgovski S.G., Pahre M.A., de Carvalho R.R., 1995, Fresh Views of Elliptical Galaxies, Buzzoni et al. (eds.) ASP Conf. Ser. 86, 129

D’Onofrio M., Capaccioli M., Zaggia S.R., Caon N., 1997, MNRAS 289, 847

D’Onofrio M., Zaggia S.R., Longo G., Caon N., Capaccioli M., 1995, A\&A 296, 319 (D95)
Dressler A., Lynden-Bell D., Burstein D., et al., 1987, ApJ 313, 42

Ferguson H.C., 1989, AJ 98, 367

Fisher D., 1997, The Second Stromlo Symposium: The Nature of Elliptical Galaxies, Arnaboldi et al. (eds.) ASP Conf. Ser. 116, 111

Forbes D.A., Franx M., Illingworth G., 1995, AJ 109, 1988

Franx M., Illingworth G.D., Heckman T., 1989, ApJ 344, 613

Galletta G., 1996, "Barred Galaxies", IAU Coll. 157, Buta R., Elmegreen B.G., Crocker D.A. (eds.), ASP Conf. Ser., 429

Graham A.W., Colless M.M., 1997, MNRAS 287, 221

Graham A.W., Lauer T.R., Colless M.M., Postman M., 1996, ApJ 465, 534

Illingworth G., 1983, IAU Symp. 100, Athanassoula E. (ed.). Reidel: Dordrecht, p. 257

Jørgensen I., Franx M., Kjaergaard P., 1996, MNRAS 280, 167

Kelson D.D., van Dokkum P.G., Franx M., et al., 1997, ApJ 478, L13

Kormendy J., Djorgovski S., 1989, ARA\&A 27, 235

Kormendy J., Bender R., 1996, ApJ 464, L119

Lynden-Bell D., Faber S.M., Burstein D., et al., 1988, ApJ 326, 19

Longo G., Zaggia S.R., Busarello G., Richter G., 1994, A\&AS 105,433

Mathews W.G., 1988, AJ 95, 1047

McElroy D.B., 1995, ApJS 100, 105

Michard R., Marchal J., 1994, A\&AS 105, 481

Prugniel Ph., Simien F., 1994, A\&A 282, L1

Prugniel Ph., Simien F., 1996, A\&A 309, 749

Prugniel Ph., Simien F., 1997, A\&A 321, 111

Richstone D.O., Tremaine S., 1986, AJ 92, 72

Richter G., Longo G., Lorenz H., Zaggia S., 1992, Messenger 68,48

Rix H.W., White S.D.M., 1990, ApJ 362, 52

Saglia R.P., Burstein D., Baggley G., et al., 1997, MNRAS 292, 499

Scorza C., Bender R., 1996, IAU Symp. 171, 55

Seifert W., Scorza C., 1996, A\&A 310, 75

Simien F., de Vaucouleurs G., 1986, ApJ 302, 564

Sparke L.S., Sellwood J.A., 1987, MNRAS 225, 653

Statler T.S., 1991, AJ 102, 882 (a)

Statler T.S., 1991, ApJ 382, L11 (b)

Statler T.S., Dutta S.N., Bak J., 1997, The Second Stromlo Symposium: The Nature of Elliptical Galaxies, Arnaboldi et al. (eds.) ASP Conf. Ser. 116, 75

Stiavelli M., Møller P., Zeilinger W.W., 1993, A\&A 277, 421

Tonry J.L., 1983, ApJ 283, L27

Van der Marel R.P., Franx M., 1993, ApJ 407, 525

van Dokkum P.G., Franx M., 1996, MNRAS 281, 985

Wozniak H., Pfenninger D., 1997, A\&A 317, 14

\section{Appendix A: Kinematic profiles}

Velocity dispersion profiles (VDPs) and rotation curves (RCs) for the 12 galaxies. The major-axis PA points from left to right in the figures, that is, the right hand side in the plots corresponds to the easterly half of the galaxies major-axis. 


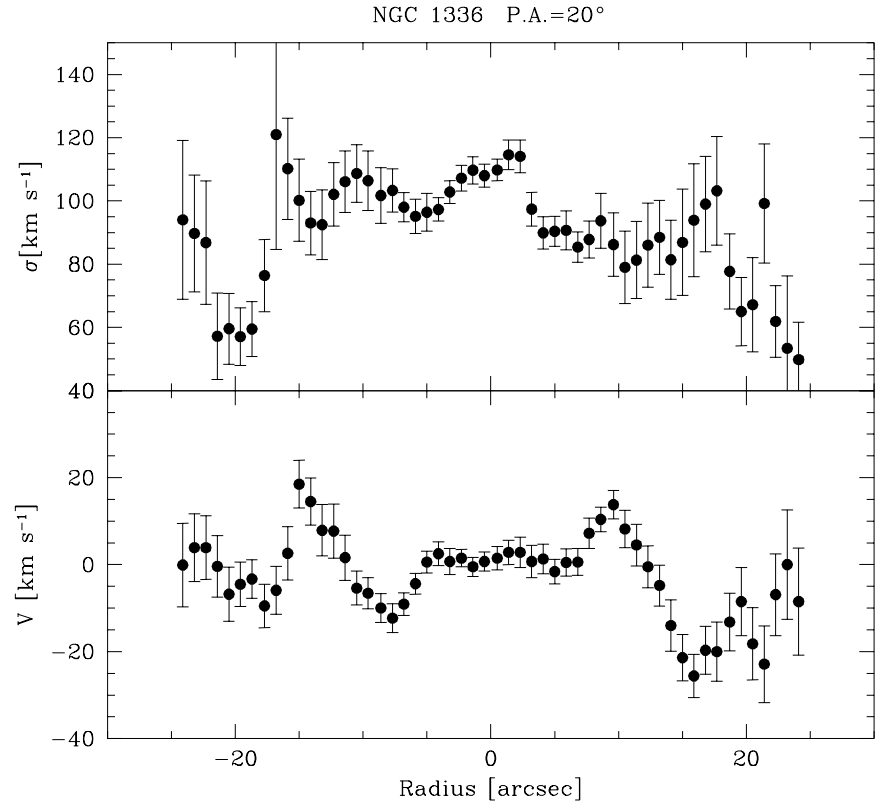

Fig. A1. NGC 1336

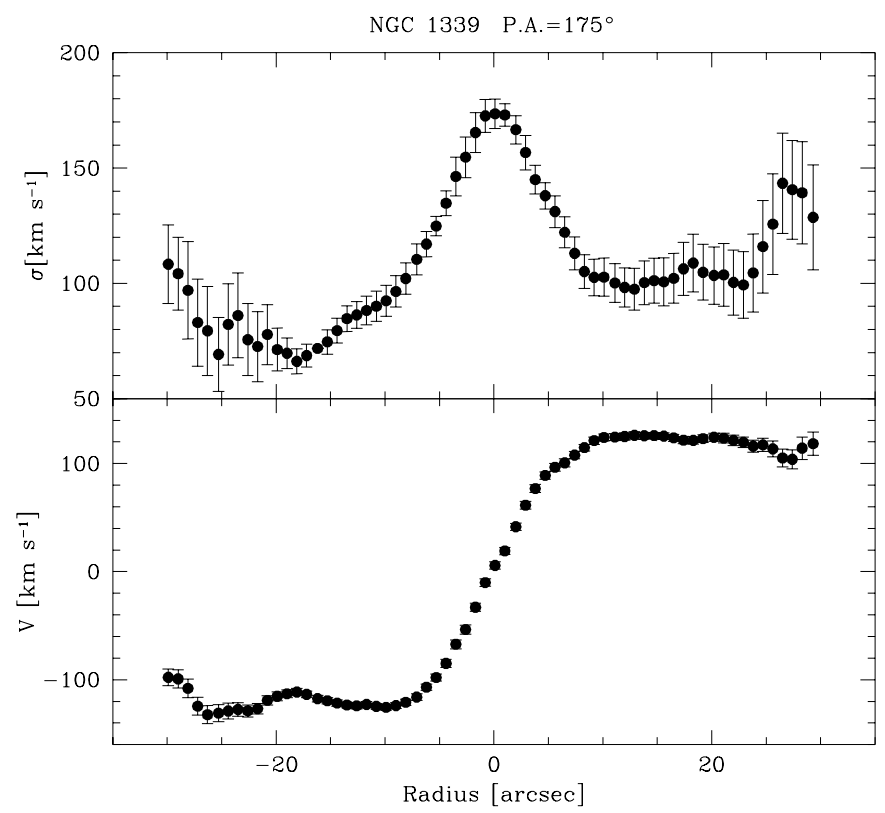

Fig. A2. NGC 1339

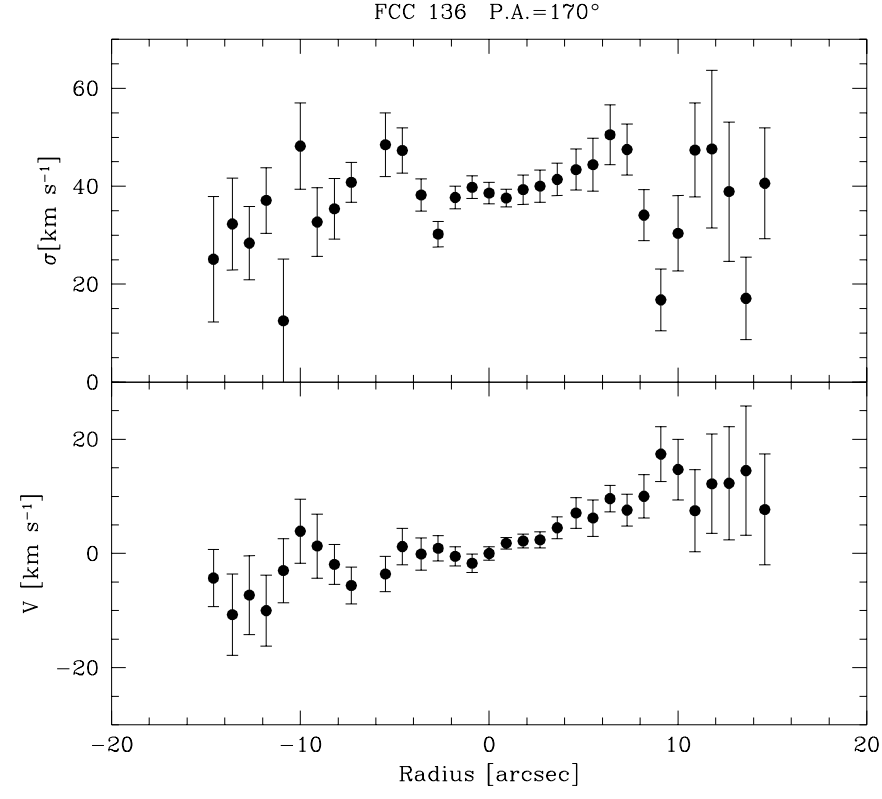

Fig. A3. FCC 136

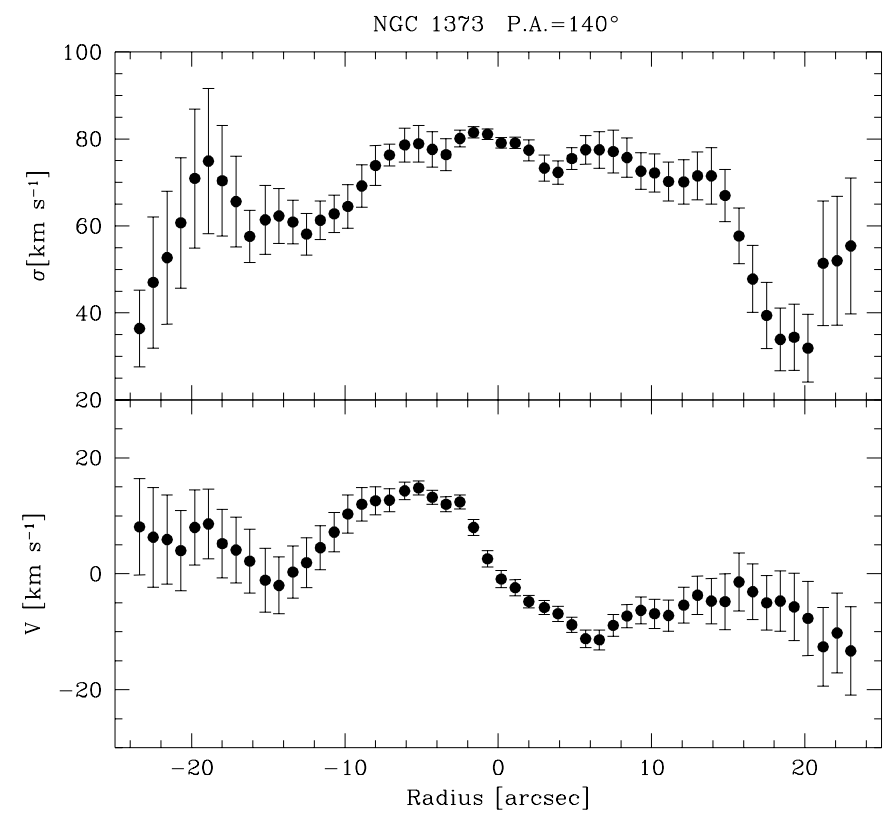

Fig. A4. NGC 1373 


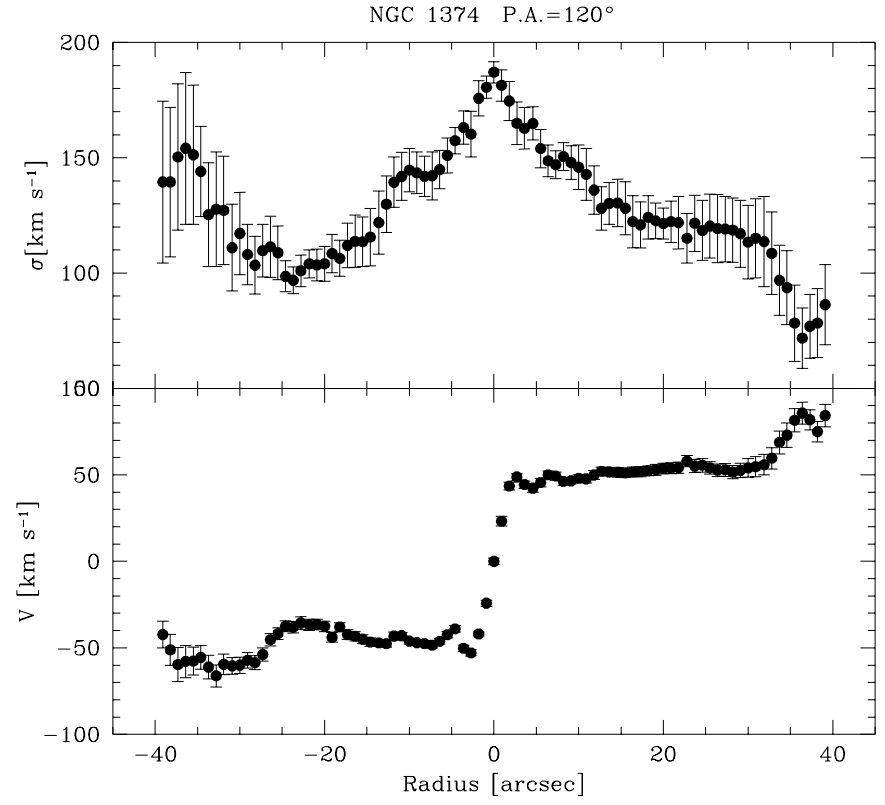

Fig. A5. NGC 1374

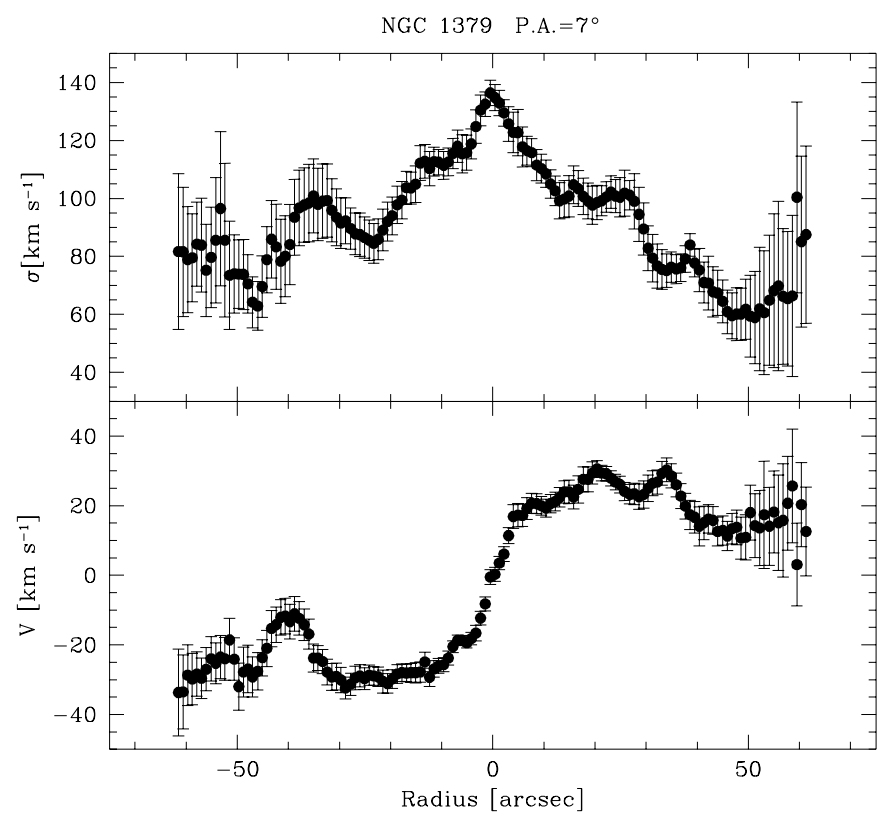

Fig. A6. NGC 1379

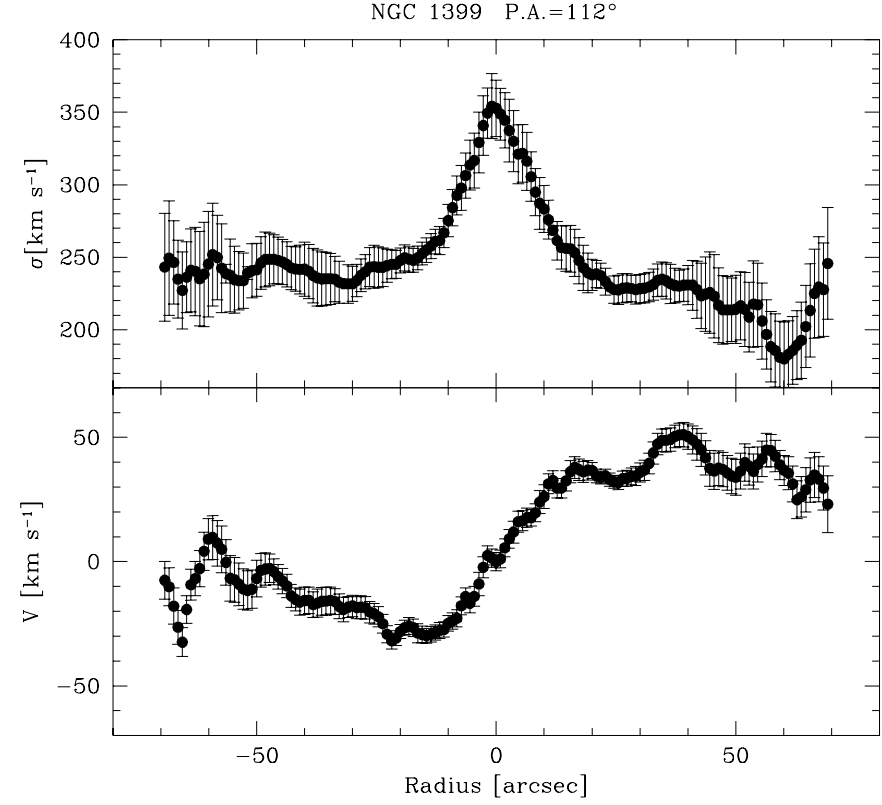

Fig. A7. NGC 1399

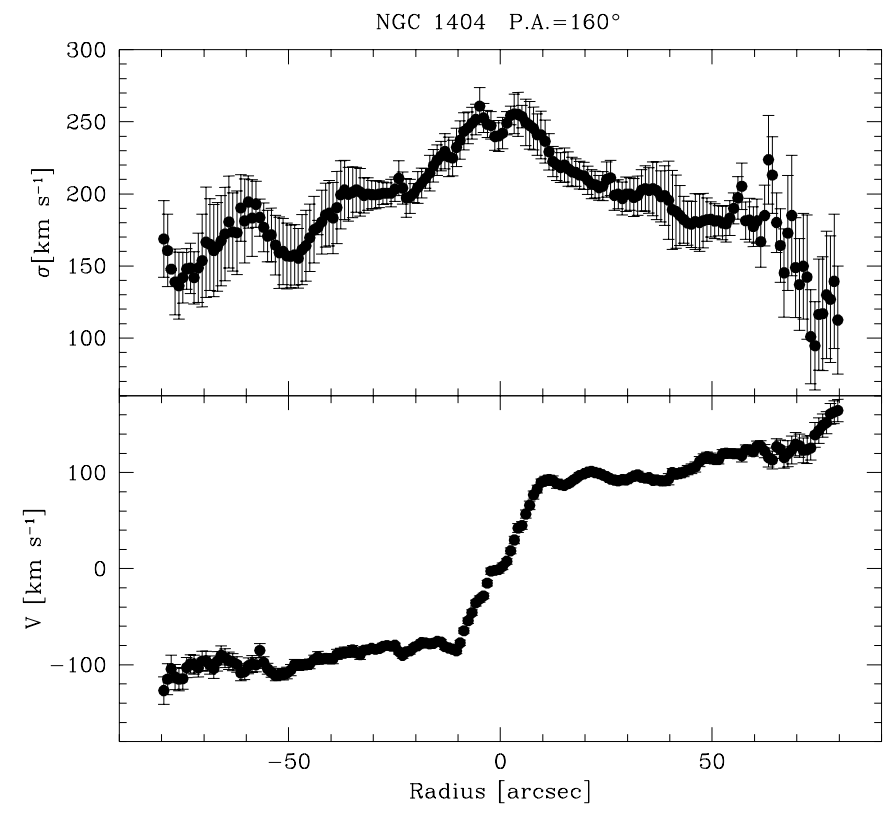

Fig. A8. NGC 1404 


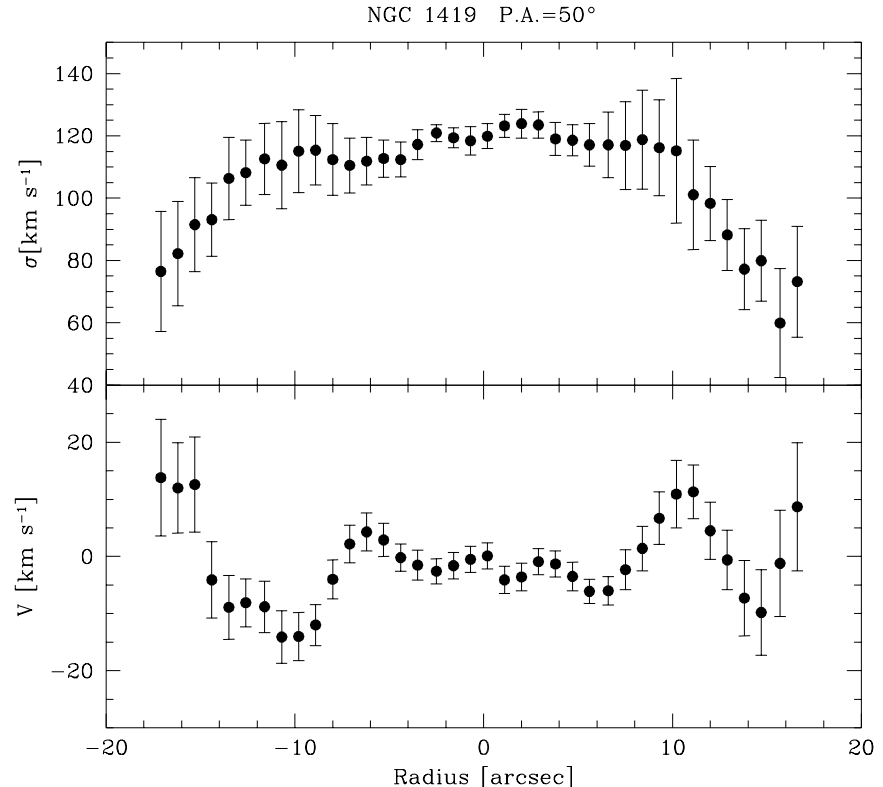

Fig. A9. NGC 1419

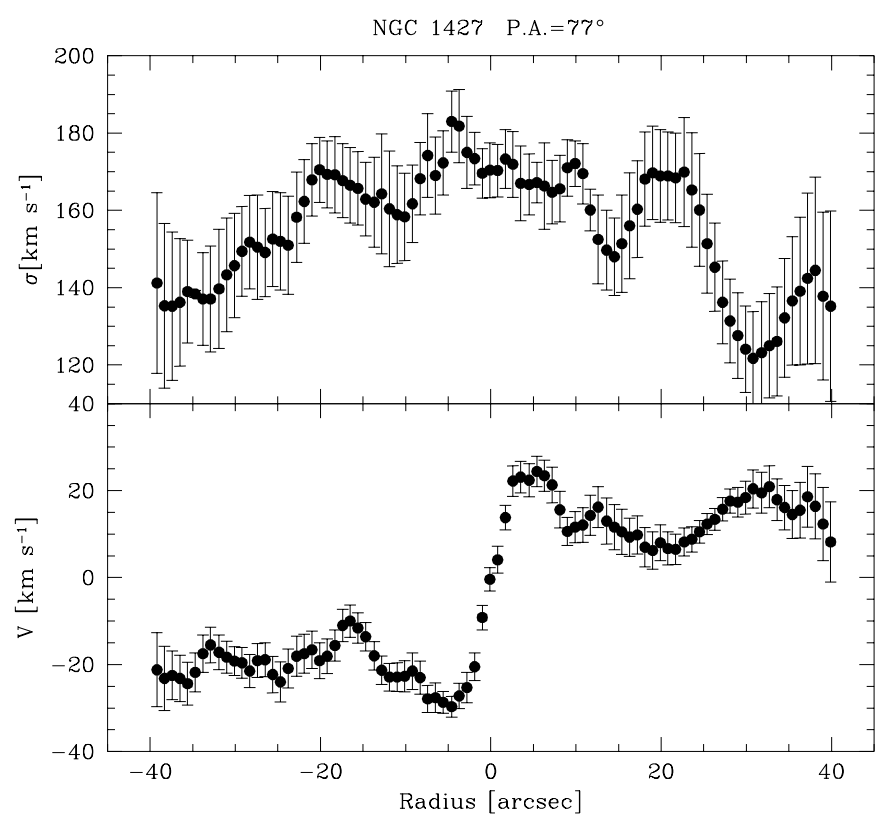

Fig. A10. NGC 1427

\section{Appendix B: Literature comparison of kinematic profiles}

Comparison with previous data. Upper panels: velocity dispersion, lower panels: rotation curve. Different sources are marked with different symbols as explained in the captions. Notice that, in general, the PA of different authors do not exactly coincide. Those which are different from ours by more than $5^{\circ}$ are the following: Bicknell et al.

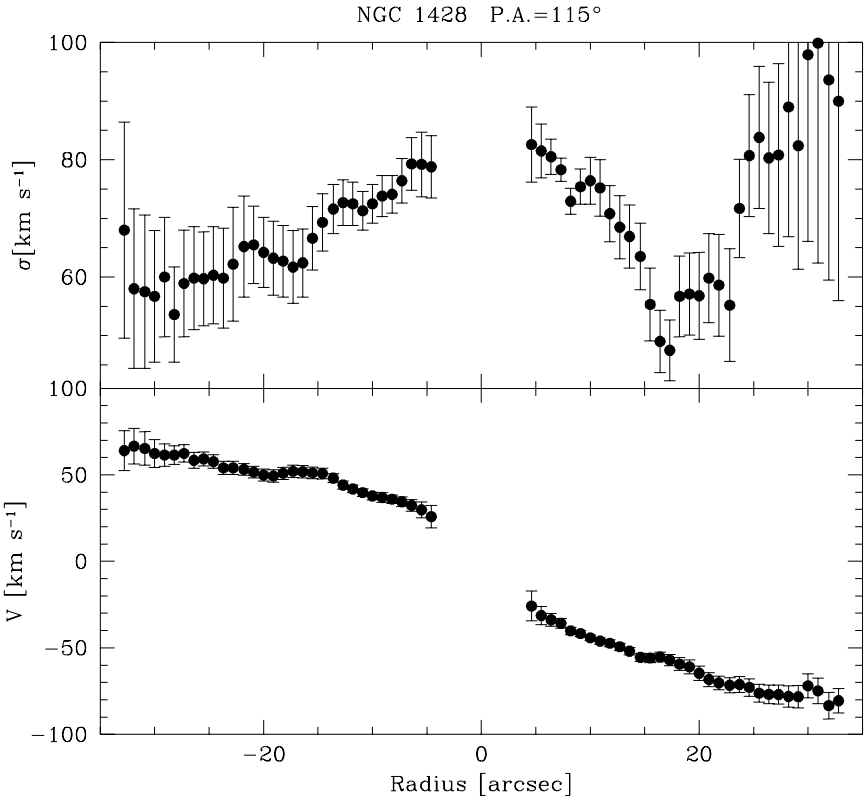

Fig. A11. NGC 1428

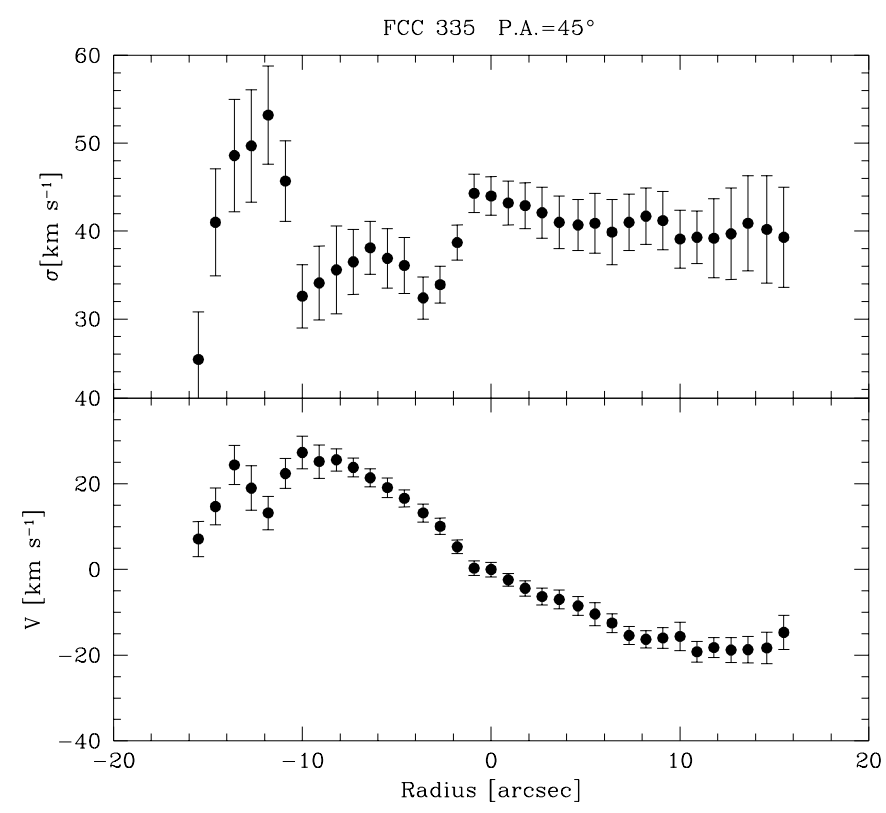

Fig. A12. FCC 335
(1989) and Stiavelli et al. (1993) adopted PA $=84^{\circ}$ for NGC 1399 (instead of $112^{\circ}$ ); van der Marel \& Franx (1993) adopted $\mathrm{PA}=110^{\circ}$ for $\mathrm{NGC} 1374$ (instead of $120^{\circ}$ ); D95 adopted $\mathrm{PA}=65^{\circ}$ for $\mathrm{NGC} 1419$ (instead of $50^{\circ}$ ). 

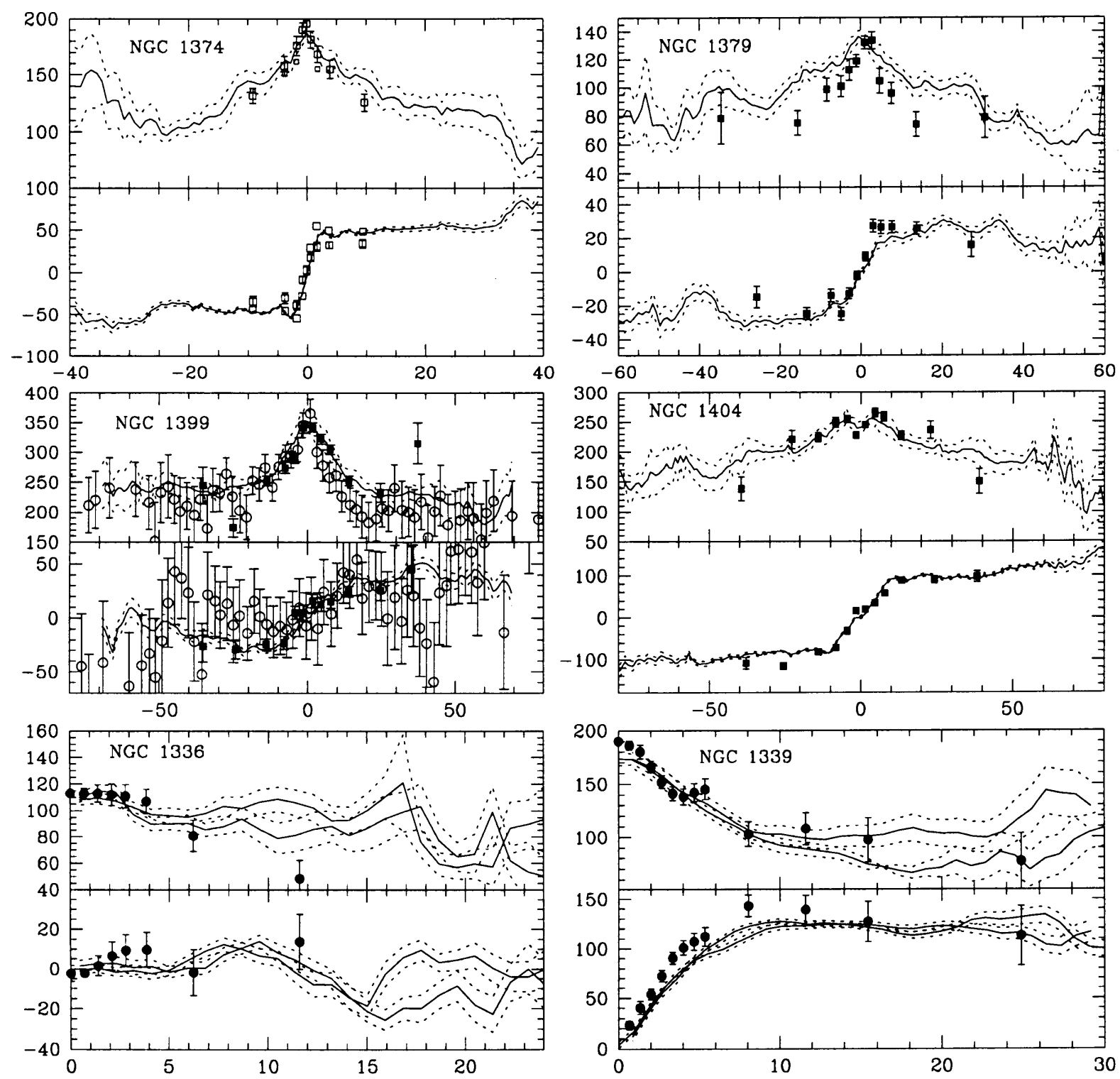

Fig. B1. Comparison with previous data. Upper panels: velocity dispersion, lower panels: rotation curve. The radial scale is in arcseconds. The continuous lines represent the present data, while the errors are marked by the dashed lines. Open boxes: Van der Marel \& Franx (1993); filled boxes: Franx et al. (1989); open circles: Bicknell et al. (1989); filled dots: D'Onofrio et al. (1995) 

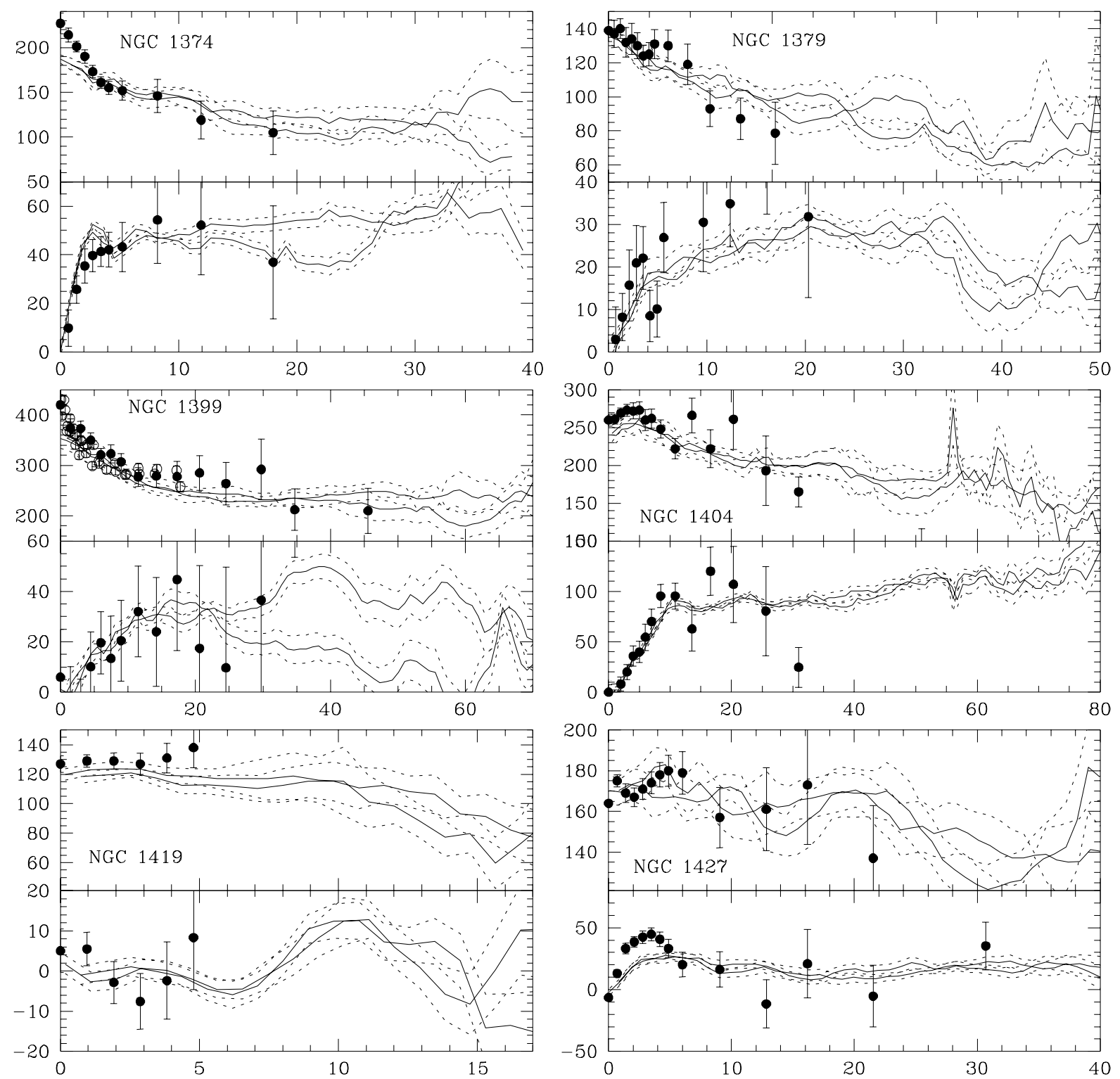

Fig. B2. As in Fig. B1. Filled circles: D'Onofrio et al. (1995); open circles: Stiavelli et al. (1993) 